\title{
1. Allgemeine Einleitung
}

Ludwig Wittgenstein (1889-1951) gilt heute als ein Klassiker der Philosophie, und er gehört wohl zu den am intensivsten diskutierten Denkern des 20. Jahrhunderts. Maßgeblichen Anteil an dieser breiten Rezeption, aber auch an dem immensen Einfluß, den sein Denken bis heute ausübt, haben vor allem zwei seiner Werke: Zum einen sein früh verfaßter und im Jahr 1921 erstmals publizierter „Tractatus logico-philosophicus“ (fortan: TLP), zum anderen seine Spätschrift „Philosophische Untersuchungen“ (im weiteren: PU) ${ }^{1}$, deren letzte Fassung ab den 40er Jahren entsteht und die im Jahr 1953 postum erscheint - wie die meisten seiner weiteren Werke. Diese sind oft nicht von Wittgenstein fertiggestellt und von den Verwaltern seines Nachlasses ediert worden.

Zentral sind für Wittgenstein vor allem Überlegungen zur Sprachphilosophie, wovon die beiden genannten Bücher auf unterschiedliche Weise Zeugnis ablegen. Darüber hinaus hat Wittgenstein aber auch auf einer Vielzahl anderer philosophischer Gebiete gearbeitet. $\mathrm{Zu}$ nennen sind in diesem Zusammenhang seine Arbeiten zur Philosophie der Mathematik und zur formalen Logik, zur Philosophie des Geistes (,Über die Philosophie der Psychologie") und zur Erkenntnistheorie (unter anderem: Wahrnehmungstheorie). Die Epistemologie im Kontext sprachphilosophischer Überlegungen hat Wittgenstein ebenfalls breit erörtert, vor allem ist hier sein aus dem Nachlaß herausgegebener Text „Über Gewißheit“ (fortan: ÜG) anzuführen, der insbesondere in den letzten drei Jahrzehnten mit in den Mittelpunkt der Wittgenstein-Forschung gerückt ist. Ferner gibt es von Wittgenstein einige wenige Überlegungen zur Ethik ${ }^{2}$ und, einige mehr, zur Philosophie der Religion sowie zur Ästhetik.

Der TLP und die PU unterscheiden sich formal beträchtlich (wie im folgenden noch deutlich werden wird), und ihr inhaltliches Verhältnis zuein-

${ }^{1}$ Die „Philosophischen Untersuchungen“, Teil I, werden nachfolgend, wie üblich, nach Paragraphen zitiert; lediglich bei den Zusätzen wird auf die Seitenzahlen der Suhrkamp-Ausgabe verwiesen. Abweichungen der gängigen Ausgaben zu der neuen genetisch-kritischen Ausgabe werden mit PU-KGE vermerkt.

${ }^{2}$ Vgl. hierzu Kellerwessel, W.: Normenbegründung in der analytischen Ethik, Kap. 1.1 . 
ander ist in der Forschung bis heute umstritten: Einige WittgensteinExegeten betonen die von ihnen angenommenen Kontinuitäten, andere die von ihnen gesehenen Diskontinuitäten bzw. unterstreichen eine unterstellte signifikante Eigenkritik und -korrektur Wittgensteins.

Betrachtet man die Rezeption beider Werke, lassen sich zumindest beträchtliche Unterschiede feststellen: Der TLP beeinflußte in hohem Maße B. Russell und den Wiener Kreis (vor allem M. Schlick und R. Carnap). Damit trägt dieses frühe Buch Wittgensteins wesentlich zur „,sprachphilosophischen Wende“ im 20. Jahrhundert bei und beeinflußt die sog. „formalsprachliche" Richtung der Analytischen Philosophie (die auf eine Konstruktion einer idealen Sprache abzielte) ${ }^{3}$. Zugleich damit leistet es einen nennenswerten Beitrag zur Verbreitung der Analytischen Philosophie im deutschsprachigen Raum. Allerdings bricht diese Entwicklung in Deutschland 1933 und in Österreich 1938 aufgrund der drastischen politischen Änderungen ab (Hitler wird Reichskanzler bzw. Österreich dem Deutschen Reich angeschlossen). Die Gründe für diesen Bruch in der Entwicklung sind einerseits darin zu sehen, daß Menschen mit jüdischen Vorfahren unter den Vertretern der Analytischen Philosophie waren, und andererseits, daß eine von der Analytischen Philosophie angestrebte Erkenntnis, die in der Tradition der Aufklärung steht, zur nationalsozialistischen Ideologie in krassestem Gegensatz steht. Durch die Emigration zahlreicher analytischer Philosophen insbesondere in die USA setzt sich die Tradition dort allerdings fort, und ist insbesondere dank W. Stegmüller in den deutschsprachigen Raum zurückgekehrt. Wittgenstein selbst (vgl. die biographische Skizze im Anhang) verbringt die Vorkriegszeit und die Zeit des Zweiten Weltkriegs überwiegend in Großbritannien, und hier entfaltet er zunehmend seine Wirkung.

Maßgeblichen Anteil daran haben auch seine Manuskripte der „mittleren Zeit", also diejenigen, die die nach dem TLP entstehen und sprachphilosophische Überlegungen entfalten, die sich auf die in den PU vertretenen Ansichten zubewegen ${ }^{4}$ : Diese Manuskripte bzw. Typoskripte zirkulieren in Abschriften und wirken auf die englische Sprachphilosophie. Verstärkt

\footnotetext{
3 Aufschluß über die philosophischen Auseinandersetzungen zwischen Wittgenstein und dem Wiener Kreis geben auch die publizierten Gespräche: Wittgenstein und der Wiener Kreis.

${ }^{4}$ Vgl. hierzu z.B. Kenny, A.: Wittgenstein, Kap. 8.
} 
wird dieser Trend dann später zweifelsohne durch die PU, die maßgeblich eine Wende zur Pragmatik einleiten und die sog. „Philosophie der normalen Sprache" prägen. ${ }^{5} \mathrm{Zu}$ nennen sind hier zunächst, geht es um direkte Einflüsse, Wittgensteins „Schüler“ (als Schüler im engeren Sinne gelten u.a.: G.E.M. Anscombe, G.P. Baker, M. Black, P.M.S. Hacker, A. Kenny, N. Malcolm, R. Rhees, F. Waismann, P. Winch, J. Wisdom). Aber auch andere wichtige Denker wurden wohl im Laufe der Zeit mehr oder weniger stark von der Wittgensteinschen Philosophie angeregt, beeinflußt oder gar (mit-)geprägt: $\mathrm{Zu}$ erwähnen sind beispielsweise K.-O. Apel, J.L. Austin, R. Brandom, M. Dummett, P.Th. Geach, R. Haller, R.M. Hare, J. Hintikka, J. McDowell, W.V.O. Quine, R. Rorty, G. Ryle, E. von Savigny, J.R. Searle, W. Sellars, P.F. Strawson, W. Stegmüller, A. Stroll, St.E. Toulmin und G.H. von Wright. Darüber hinaus entfaltete sich eine beachtliche Wirkung des späten Denkens von Wittgenstein auch außerhalb der Philosophie, nämlich in der Theologie bzw. Religionswissenschaft (J. Hick, D.Z. Phillips) und in der Soziologie (P. Winch).

Nicht unerwähnt bleiben soll, daß nicht wenige namhafte analytische Philosophen als bedeutsame Kritiker der späten Wittgensteinschen Sprachphilosophie anzusehen sind, wie beispielsweise B. Russell, S.A. Kripke, H. Putnam oder J.J. Katz. Und nicht verschwiegen werden sollte, daß gleichfalls bedeutende Kritiker der späten Philosophie von Wittgenstein auch von außerhalb der analytischen Philosophie gekommen sind, wie der kritische Rationalist K. Popper oder der Neomarxist H. Marcuse. ${ }^{6}$

Wie unterschiedlich die Einschätzungen des Wertes der Sprachphilosophie der PU selbst innerhalb der analytischen Philosophie sind, verdeutlichen folgende kurze Stellungnahmen. Betont kritisch hat sich B. Russell über die späte Philosophie Wittgensteins geäußert:

\footnotetext{
${ }^{5}$ Die Ursprünge dieser Strömung der Analytischen Philosophie reichen allerdings bis zu deren Anfängen zurück, genauer bis zur frühen Philosophie des G.E. Moore. Weitere wichtige Vertreter sind beispielsweise G. Ryle und P.F. Strawson.

${ }^{6}$ Vgl. zur Wirkung Wittgensteins im Rahmen der Analytischen Philosophie, vor allem aber auch zu Ähnlichkeiten mit und Unterschieden zu anderen analytischen Philosophen wie Quine, Strawson, Ryle u.a.: Hacker, P.M.S.: Wittgenstein im Kontext der analytischen Philosophie.
} 
„In Wittgensteins Philosophischen Untersuchungen habe ich nichts entdeckt, was mein Interesse weckt, und es bleibt mir unbegreiflich, wieso eine ganze Richtung der Philosophie auf den Seiten des Buches wichtige Weisheiten zu erkennen glaubt. [...] Wenn er Recht hat, ist die Philosophie bestenfalls eine geringfügige Hilfe für Lexikographen, schlimmstenfalls eine müßige Unterhaltung am Teetisch." ${ }^{\text {"7 }}$

$\mathrm{Da}$ diese ausgesprochen negativ wertende Einschätzung auf einem (auch von anderen Wittgenstein-Kritikern geteilten) Mißverstehen der Methode Wittgensteins beruht, wird im folgenden deutlich. Denn eine bloße Hilfe für die Erstellung von Wörterbüchern ist zweifelsohne nicht das Resultat der Sprachphilosophie der PU.

Sehr viel positiver ist hingegen die Auffassung von Ryle, der meint: „Wittgenstein hat dafür gesorgt, daß unsere Philosophengeneration die Philosophie selbst problematisiert", und der darüber hinaus die Auffassung vertreten hat: „Vor allem haben wir gelernt, aufmerksam darauf zu achten, was man sagen und was man nicht sagen kann“"8. Damit zeigt sich, daß Ryle zwei zentrale Anliegen des späten Wittgensteins angemessen erfaßt: Erstens festzustellen, was sich sinnvoll sagen läßt und was sich nicht sinnvoll sagen läßt - eine Frage, die sich auch die frühere Philosophie des Wiener Kreises gestellt hat und die der TLP zu beantworten gesucht hat. Und zweitens zu klären, was überhaupt eine sinnvolle Frage der Philosophie sei, welche Probleme durch sie also ernsthaft zu beantworten versucht werden kann, und welche Fragen zurückzuweisen sind.

Diesen Punkt spricht auch Strawson an, der Wittgenstein bzw. den Verfasser der PU für einen ,genialen Philosophen“ hält:

„Er [Wittgenstein] besitzt ein außerordentliches, beinahe einzigartiges Vermögen zur Vertreibung philosophischer Illusionen, und er hilft uns, deutlich zu sehen, wie die Sprache - und folglich unser Denken - wirklich funktioniert. [...] Es dürfte schwer fallen, einen anderen Philosophen zu nennen, der wahrscheinlich einen so tiefreichenden und bleibenden Einfluß haben wird.“9

Die Vielfalt an Deutungen und Einordnungen der PU geht über das Gesagte allerdings weit hinaus. Bereits im Jahre 1967 hat Hans Lenk einen breiteren Überblick über diese gegeben, der Charakterisierungen der PU als „verborgener metaphysischer Realismus“, ,antirealistischer ,linguistisch-

\footnotetext{
${ }^{7}$ Russell, B.: Philosophie. Die Entwicklung meines Denkens, S. 224.

${ }^{8}$ Ryle, G.: Ludwig Wittgenstein, S. 256; deutsch nach Hacker 1997, S. 266.

${ }^{9}$ Strawson über Wittgenstein in einem Interview der Zeitschrift „Cogito“, wieder abgedruckt in Pyle, A. (Hg.): Key Philosophers in Kontext, S. 42; deutsch nach Hacker 1997, S. 266.
} 
pragmatischer Nominalismus'“, „Panlinguismus“, „Behaviorismus“, „Vollblut-Konventionalismus“, und „philosophischer Konstruktivismus“ enthält, des weiteren aber auch die behauptete und bestrittene Nähe zur Phänomenologie anführt, einen „metaphysischen Skeptizismus“ ebenso verzeichnet wie das Etikett „Transzendentalphilosoph“, so daß Wittgenstein ,als Vertreter eines synthetischen Apriorismus“ " erscheint. $^{10}$

In Anbetracht der Einschätzung, Wittgenstein sei ein Klassiker der Philosophie, also höchst bedeutsam, und in Anbetracht der immensen Deutungsvielfalt und damit einhergehenden Uneinigkeit, drängt es sich auf, eine sorgsame, Schritt für Schritt verfahrende, ausgesprochen textnahe Interpretation zu erarbeiten, die dann auch eine zuverlässige Basis für weitergehende Einschätzungen sein kann. Sie soll versuchen, Wittgensteins außerordentlich exakter Untersuchung des sinnvollen Sprachgebrauchs gerecht zu werden, und so zugleich seinem Anliegen, damit auch den Bereich des sinnvollen Denkens auszuloten. Schließlich, darin besteht wohl eine deutlichere bzw. weitergehende Einstimmigkeit, befaßt sich Wittgenstein in den PU mit durchaus wichtigen Fragestellungen der Philosophie.

${ }^{10}$ Vgl. hierzu Lenk, H.: Zu Wittgensteins Theorie der Sprachspiele, S. 57 und 133. 
